\title{
Ballistic and localized transport for the atom optics kicked rotor in the limit of vanishing kicking period
}

\author{
Mark Sadgrove, ${ }^{1}$ Sandro Wimberger, ${ }^{2}$ Scott Parkins, ${ }^{1}$ Rainer Leonhardt ${ }^{1}$ \\ ${ }^{1}$ Department of Physics, University of Auckland, Private Bag 92019, Auckland, New Zealand \\ ${ }^{2}$ Dipartimento di Fisica E. Fermi, Università di Pisa, Largo Pontecorvo 3, 56127 Pisa, Italy
}

\begin{abstract}
We present mean energy measurements for the atom optics kicked rotor as the kicking period tends to zero. A narrow resonance is observed marked by quadratic energy growth, in parallel with a complete freezing of the energy absorption away from the resonance peak. Both phenomena are explained by classical means, taking proper account of the atoms' initial momentum distribution.
\end{abstract}

PACS numbers: 05.45.Mt, 42.50.Vk, 32.80.Qk,05.60.-k

The atom optics realization of the paradigmatic kicked rotor (KR) [1] allows the experimental study of uniquely quantum mechanical aspects of a fundamental, classically nonlinear system. Dynamical localization is perhaps the most celebrated quantum phenomenon observed in the quantum KR 1, 2], however the phenomenon of quantum resonance, whereby some atoms experience enhanced energy growth (quadratic in kick number) for well-defined kicking periods, has received renewed theoretical and experimental attention of late.

The possibility for quadratic energy growth to occur for the KR at quantum resonance has been known for some time [3]. The first experiments to observe phenomena related to quantum resonance employed the atom optics kicked rotor (AOKR) to study the momentum distributions of ensembles of kicked rotors 4], although the broad initial momentum distributions involved prohibited the observation of quadratic mean energy growth. Recent studies have shown that the quantum resonances, initially thought to be extremely sensitive quantum effects, can be explained by purely classical means [5], and that, indeed, only linear growth in mean energy is expected at quantum resonance for experiments starting with a broad initial momentum distribution 5]. The experimental signatures of quantum resonance behaviour prove to be robust with respect to decoherence by spontaneous emission [5, [6], amplitude noise [7, 8] , and also to large perturbations of the kicking strength 9 ]. These counterintuitive findings make the quantum resonances a potential source for creating directed fast atoms, e.g. for the study of quantum random walks [10]. However, even for narrow initial atomic momentum distributions (i.e. those with standard deviation $\sigma_{p}<$ two-photon recoils), true ballistic mean energy growth has only been observed convincingly for up to two kicks [11].

In this letter, we present the first signatures of true ballistic peaks in the mean energy of a kicked atomic ensemble, with a relatively broad initial momentum distribution, but for an essentially classical regime. Our seemingly counterintuitive experimental results are explained by the same technique as used to classically describe the structure of the quantum resonance peaks of the AOKR
5]. Additionally, we observe a regime in which an unexpected dynamical freezing effect occurs, which corresponds to atoms ceasing to absorb energy from the kicks. The close proximity, in terms of pulse period, of the regimes of ballistic and frozen energy growth promises to allow fine control of atomic velocities.

We realize the AOKR by subjecting cold Cesium atoms to a far detuned standing wave with spatial period $\pi / k_{L}$ ( $k_{L}$ being the wave number of the kicking laser) and pulsed with period $T$. Our system is described, in dimensionless units, by the Hamiltonian [14]

$$
\mathcal{H}\left(t^{\prime}\right)=\frac{p^{2}}{2}+k \cos (x) \sum_{t=0}^{N} \delta\left(t^{\prime}-t \tau\right),
$$

where $p$ is the atomic momentum in units of $2 \hbar k_{L}$ (i.e. of two-photon recoils), $x$ is the atomic position divided by $2 k_{L}, t^{\prime}$ is time and $t$ is an integer which counts the kicks. Experimentally, we approximate $\delta$-kicks by pulses of width $\tau_{p}$ which are approximately rectangular in shape. We also define an effective Planck's constant $\tau=8 \omega_{r} T$, where $\omega_{r}$ is the recoil frequency (associated with the energy change of a Caesium atom after emission of a photon with $k_{L}=7.37 \times 10^{6} \mathrm{~m}^{-1}$ ). The dimensionless parameter $k$ is the kicking strength of the system. By exploiting the spatial periodicity of the Hamiltonian, we can use Bloch's theorem to reduce the atomic dynamics along the $\mathrm{x}$ axis to that of a rotor on a circle, as described in [5]. Then, the one-kick propagator for a given atom is

$$
\hat{\mathcal{U}}_{\beta}=e^{-\mathrm{i} k \cos (\hat{\theta})} e^{-\mathrm{i} \tau(\hat{\mathcal{N}}+\beta)^{2} / 2},
$$

where $\theta=x \bmod (2 \pi), \hat{\mathcal{N}}=-\mathrm{i} d / d \theta$, and $\beta$ is the quasimomentum (i.e., the non-integer part of $p$ ). To examine the limit as $\tau \rightarrow 0$, we can use the classical version of the mapping induced by the Hamiltonian (1). We define $J=\tau p$ and find

$$
J_{t+1}=J_{t}+\tilde{k} \sin \left(\theta_{t+1}\right), \theta_{t+1}=\theta_{t}+J_{t},
$$

where $\tilde{k} \equiv \tau k$ is the classical stochasticity parameter of the standard map [12]. (3) implicitly contains the dif- 


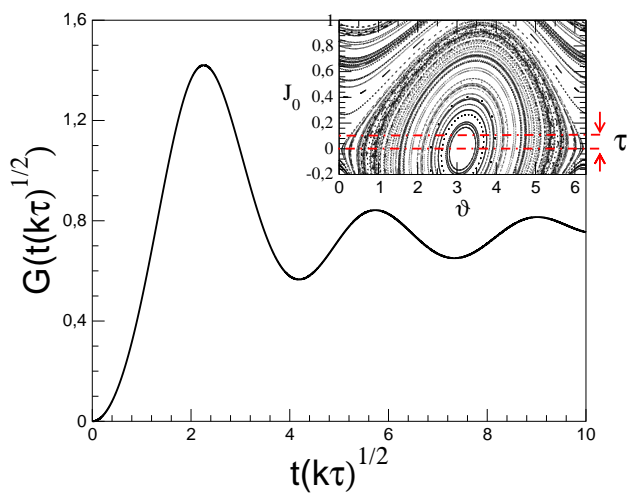

FIG. 1: The function $G$ vs. $x=t(k \tau)^{1 / 2}$ for $J_{0} \in[0,0.1)$. The inset shows a portion of the classical phase space of the map (3), for $\tau=0.1, k=2.5$. The dashed lines mark the position of the initial momenta, corresponding to a uniform distribution of quasimomenta in $[0,1)$.

ferent quasimomentum classes which are vital to the description of quantum resonances [5]. In fact, (3) is equivalent to the $\epsilon$-classical standard map developed in [5] to describe quantum resonance behaviour when $\tau=2 \pi l+\epsilon$ for the special case where $l=0$. Below, we will see that, in our case, the typically uniform initial distribution of quasimomenta [5, 6] does not hinder ballistic motion at the observed resonance as $\tau \rightarrow 0$.

We now compute the mean energy of an ensemble of kicked atoms from Eq. (3). Assuming for simplicity an initially flat distribution of $p \in[0,1)$ we have $J_{0}=\tau \beta_{0}$ with $\beta_{0}$ uniformly distributed in $[0,1)$. Since $J=\tau p$, the mean energy of the rotor at time $t$ is given by $\left\langle E_{t, \tau}\right\rangle=\tau^{-2}\left\langle\left(\delta J_{t}\right)^{2}\right\rangle / 2, \delta J_{t}=J_{t}-J_{0}$. As the initial conditions in phase space populate mainly the region close to $J_{0}=0$, we can compute $\left\langle E_{t . \tau}\right\rangle$ for $\tau>0$ by means of the pendulum approximation [12]. The motion is described (in continuous time) by $H_{\text {res }}=\frac{1}{2}\left(J^{\prime}\right)^{2}+\tau k \cos (\theta)$, with the characteristic time scale for the motion in the resonant zone $t_{\text {res }}=(k \tau)^{-1 / 2}$ [12]. We can formally remove $\tau$ from the Hamilton equations, by scaling momentum into $\bar{J}=J^{\prime} /(k \tau)^{1 / 2}$ and time into $t / t_{\text {res }}$. This gives $\left\langle\left(\delta J_{t}\right)^{2}\right\rangle=\left\langle\left(J_{t}^{\prime}-J_{0}^{\prime}\right)^{2}\right\rangle \simeq k \tau G$, for an ensemble of orbits started inside the resonant zone. The scaling function $G(x) \equiv \frac{\sqrt{k}}{2 \pi \sqrt{\tau}} \int_{0}^{2 \pi} \mathrm{d} \theta_{0} \int_{0}^{\sqrt{\tau / k}} \mathrm{~d} J_{0} \bar{J}\left(x, \theta_{0}, J_{0}\right)^{2} \simeq$ $\frac{1}{2 \pi} \int_{0}^{2 \pi} \mathrm{d} \theta_{0} \bar{J}\left(x, \theta_{0}, J_{0}=0\right)^{2}$ depends on the variable $x=$ $t(k \tau)^{1 / 2}$ and weakly on $k$ and $\tau$, in contrast to the quantum resonant case studied in [5]. In Fig. 1] $G$ is shown as a function of $x$. We see that $G$ tends to a level $\alpha \approx 0.7$ as $x \rightarrow \infty$. This is because $G$ is an average over nonlinear pendulum motions with a continuum of different periods, and therefore saturates to a constant value when the argument is larger than $\approx 1$. The dependence of $G$ on $\tau$ is negligibly small for $\tau \lesssim 1 / k$, so that practically, $G$ can be viewed as a function of the scaling parameter $x$ alone. We obtain as our final result for the mean energy $\left\langle E_{t, \tau}\right\rangle_{\mathrm{res}} \simeq \frac{\tau k}{2 \tau^{2}} G$. We can now immediately derive two interesting limits. Firstly, as we let $\tau \rightarrow 0$, the argument of $G$ becomes small, whence $G(x) \approx x^{2} / 2$ leading to the expression

$$
\left\langle E_{t, \tau \rightarrow 0}\right\rangle_{\mathrm{res}}=\frac{k^{2} t^{2}}{4} .
$$

This describes quadratic growth in mean energy which occurs as exact resonance is approached. We note again that in the case of quantum resonances, $\epsilon$-classical theory predicts only linear mean energy growth with kick number at resonance [5]. The later linear increase is induced by the contribution of most quasimomentum classes which lie outside the classical resonance island for $\tau=2 \pi \ell+\epsilon$ ( $\ell$ positive integer $)$. For $\tau \rightarrow 0$, almost all initial conditions (or quasimomenta) lie within the principal resonance island shown in the inset of Fig. 1.

Additionally, as we let $\tau$ grow large, $G$ saturates to the value $\alpha$ giving

$$
\left\langle E_{t, \tau>0}\right\rangle_{\mathrm{res}} \simeq \frac{k}{2 \tau} \alpha,
$$

for finite $\tau>0$ and $t^{2} k \gg 1 / \tau$. Within the stated parameter range, this result implies dynamical freezing the ensemble's mean energy is independent of kick number. This phenomenon is a classical effect in a system with a regular phase space (see inset of Fig. 11). It is distinct from dynamical localization which is the quantum suppression of momentum diffusion for a chaotic phase space. Experimentally, this freezing corresponds to the cessation of energy absorption from the kicks, similar to that which occurs at dynamical localization. The freezing is easily explained as the averaging over all trajectories which start at momenta close to zero, and move with different frequencies about the fixed point of the map (3).

We now turn to the experimental verification of ballistic growth and dynamical freezing. In our experiments, we measured the mean energy of an atomic ensemble as $\tau$ was varied and for different kick numbers. Details of our experimental setup may be found in Ref. [7] and are summarized below. Using a standard sixbeam magneto-optical trap, or MOT 15, we trap and cool Cesium atoms typically to below $10 \mu \mathrm{K}$. During an experiment, the trap is turned off and the atoms are subjected to pulses from an optical standing wave (created by a $150 \mathrm{~mW}$, frequency stabilized diode laser) detuned $500 \mathrm{MHz}$ from the $6 S_{1 / 2}(F=4) \rightarrow 6 P_{3 / 2}\left(F^{\prime}=5\right)$ transition of Cesium. Pulse durations of $\tau_{p}=240 \mathrm{~ns}$ and $320 \mathrm{~ns}$ were used for our experiments with optical powers of $P=20 \mathrm{~mW}$ and $30 \mathrm{~mW}$ respectively, and the atoms were subjected to at most 20 kicks. For these parameters, the effect of spontaneous emission is found to be negligible. The experimental momentum distribution of the atoms is found from a fluoresence image of the cloud using the standard time of flight technique [4, 6, 7] and the mean kinetic energy may be calculated from the second 


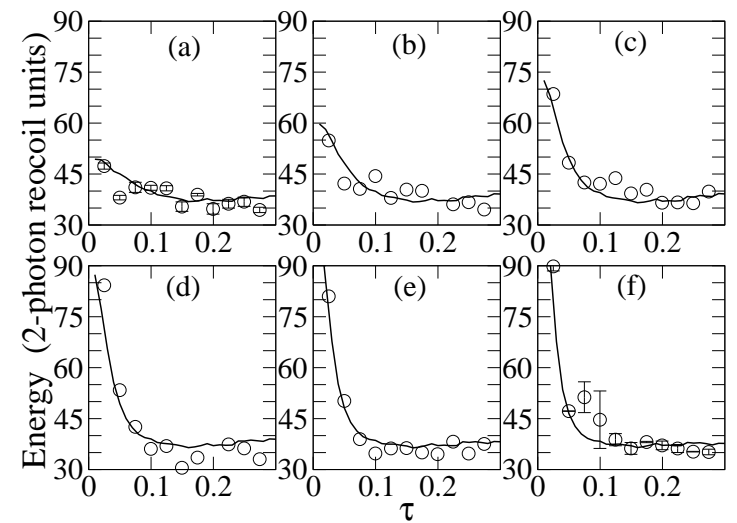

FIG. 2: Results from experiments (circles) and classical simulations (solid line) showing energy vs. $\tau$ for $k=2.5$ and $t=3,4,5,6,7,8$ for Figs. (a,b,c,d,e,f) respectively. The error bars shown in (a) and (f) correspond to statistical fluctuations over three experimental shots (they do not take into account systematic effects such as power fluctuations of the kicking laser).

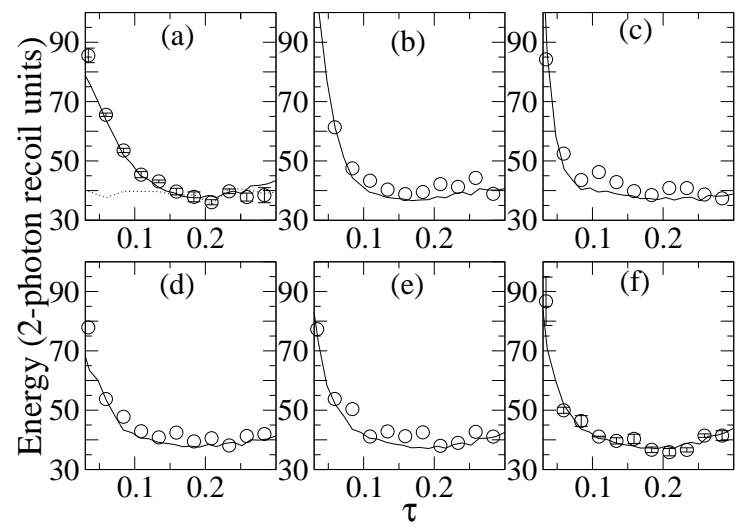

FIG. 3: Data as in Fig.2, for $k=4.9$ and $t=3,5,7,12,16,20$ for Figs. (a,b,c,d,e,f) respectively. The dotted line in (a) shows the measured energy after just 1 kick.

moment of this distribution. In order to probe the very narrow resonance predicted above as $\tau \rightarrow 0$, it was necessary to use very small pulse periods, leading to violations of the $\delta$-kick assumption. Nonetheless, we found that finite pulse duration effects were negligible for our results due to the relatively low kick strengths, kick numbers and kinetic energies involved. For moderate laser powers and kicking pulse durations, it is possible to estimate $k$ using the method employed in Ref. [16]. Here the difference in energies after one and two kicks was found for values of $\tau>1$ where the energy growth is known to be quasilinear. From the standard $\delta$-kicked rotor theory, the energy difference is $k^{2} / 4$ [12], allowing $k$ to be determined without precise knowledge of the atoms' initial thermal energy.

To predict the correct offset from zero energy of our measurements, it is still necessary to calculate the stan- dard deviation $\sigma_{p}$ of the initial experimental momentum distribution. The calculation of $\sigma_{p}$ requires truncation of the wings of the experimental momentum distribution at some momentum so that the second moment calculation is not ruined by noise in the wings. Since the initial momentum distribution has considerable non-Gaussian wings, we inevitably underestimate the value of $\sigma_{p}$ using this method. We estimate this systematic error to be less than $20 \%$ or 1.5 two-photon recoils for the experimental results presented here. Fig. 2] shows measured energies (circles) and classical data for a measured value of $k=2.5 \pm 0.1$, and various kick numbers. The classical results are obtained from the dynamics generated by (3) for a Gaussian initial ensemble of 25000 momenta $p=J / \tau$, with 200 uniformly distributed angles each. We have taken $\sigma_{p}=8.4$ for our simulations, and the results can be compared with the predictions of Eqs. (4) and (5) if the initial ensemble's energy is added to the analytic results (derived above for $p \in[0,1)$ ). Allowing for experimental uncertainties, excellent agreement is found between the measurements and our classical theory. In particular, a ballistic resonance peak is seen to exist and, although the vanishing resonant kicking period itself cannot be probed, the trend towards quadratic growth is clear in the data. The resonance is very narrow - much more so than the quantum resonances for the same parameters. Specifically, the half width of the $\tau \rightarrow 0$ peak after five kicks is $\sim 0.05$ whereas that for a quantum resonance peak for the same value of $k$ would be $\sim 0.4[5]$. The extremely fast compression of the resonance peak is characteristic of a quantum resonance which would be observed for a purely plane wave initial condition with momentum $p=0$ at $\tau=4 \pi$, for instance. Hence, the peak width observed here shrinks even faster than at the quantum resonances observed in [6], for which a sub-Fourier peak width $\propto 1 / t^{2}$ was predicted [5]. This makes the resonance at vanishing kicking period a sensitive phenomenon with the potential to be useful for making high-precision measurements. The results in Fig. 2 also demonstrate that dynamical freezing, i.e., zero net energy gain, is occurring for $\tau>0.1$ after multiple kicks. To observe this localization effect more convincingly, we chose a larger value of $k$ and measured the mean energy of our atoms for up to 20 kicks. The results, shown in Fig. 3 are for a measured value of $k=4.9 \pm 0.2$, with $\sigma_{p}$ taken to be 8 for the simulations. After 20 kicks, for $\tau>0.1$, the mean energy of the atoms has not risen above the one kick level. Again, excellent agreement is seen between experimental results and classical data. As a final test we have compared our data with quantum simulations for $k=4.9, \sigma_{p}=8$. As shown in Fig. 4 the numerics and experimental measurements agree almost perfectly within the estimated errors. On closer inspection, a hint that the classical approximation fails for large $\tau$ can be found in Fig. 4, where the classical data starts to deviate (very slightly) from the quantum data for larger $\tau \geq 1 / k$. 


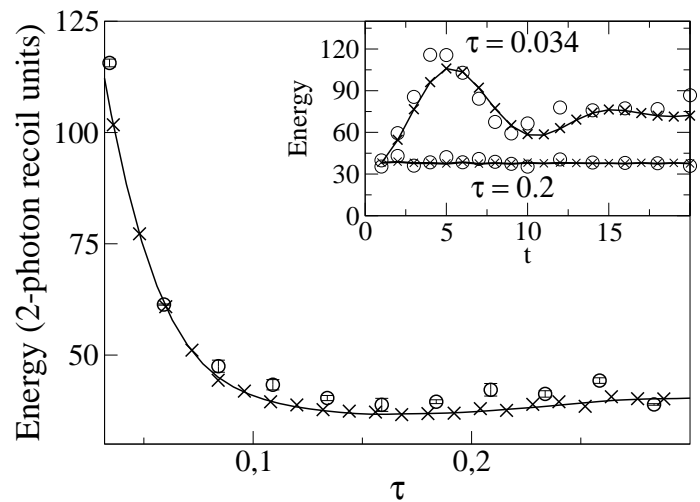

FIG. 4: Mean energy vs. $\tau$ from experiments (circles), classical (crosses) and quantum simulations (using a Gaussian initial ensemble centered at $p=0$; solid line) for $k=4.9, \sigma_{p}=8$ and $t=5$. Note the excellent agreement between experimental data and the simulations. The inset shows how the energy varies with $t$ for $\tau=0.2$ and the smallest measurable period $\tau=0.034$ (corresponding to $T=0.33 \mu \mathrm{s}$ ). We also note the immediate onset of the freezing effect for $\tau=0.2$, whilst dynamical localization would set in on a time scale much larger than 1 kick for the present kick strength [2].

Above that threshold, the standard map enters the critical regime, which mainly affects the small subclass of extremely large momenta in our initial ensemble, lying outside the principal resonance island. Stronger deviations are expected in this region for very large interaction times $(t \gg 10)$ 5]. The inset in Fig. (4 demonstrates the regimes of near resonant and off-resonant (frozen) energy growth more explicitly by plotting the mean energy against kick number. The curve for $\tau=0.034$ closely resembles the structure of the "quasi" scaling function $G$ from Fig. 1, showing both the initial ballistic growth and saturation after $t \geq 2 / \sqrt{k \tau} \simeq 5$ kicks.

In conclusion, we have experimentally observed and theoretically explained the occurrence of ballistic, resonance like transport in the limit of vanishing kicking period for a broad initial ensemble of atomic momenta. The quadratic energy growth at this resonance has been verified in comparison with the linear growth of mean energy found at quantum resonance for comparable initial conditions [6, 7]. Away from the ballistic peak, a dynamical freezing effect, as a consequence of the underlying regular classical dynamics, has been observed, corresponding to zero energy-growth rates for up to 20 kicks in our experiment. Both of these phenomena, realized here for the first time, are of great interest for experimentally controlling fast (ballistic) and slow (frozen) atomic velocities.

[1] G. Casati et. al., in Stochastic Behavior in Classical and Quantum Hamiltonian Systems, ed. by G. Casati and J. Ford (Springer, Berlin, 1979), p. 334.

[2] D.L. Shepelyansky, Physica 28D, 103 (1987); S. Fishman, in Quantum Chaos, School "E. Fermi" CXIX, eds. G. Casati et al. (IOS, Amsterdam, 1993).

[3] F.M. Izrailev and D.L. Shepelyansky Sov. Phys. Dokl. 24, 996 (1979); F.M. Izrailev, Phys. Rep. 196, 299 (1990).

[4] F.L. Moore et al., Phys. Rev. Lett. 75, 4598 (1995); W.H. Oskay et al. Opt. Comm. 179, 137 (2000).

[5] S. Wimberger, I. Guarneri, and S. Fishman, Nonlinearity 16, 1381 (2003); Phys. Rev. Lett. 92, 084102 (2004).

[6] M.B. d'Arcy et al., Phys. Rev. Lett. 87, 074102 (2001); Phys. Rev. E 69, 027201 (2004).

[7] M. Sadgrove et al., Phys. Rev. E 70, 036217 (2004).

[8] S. Brouard and J. Plata, J. Phys. A 36, 3745 (2003).

[9] S. Wimberger, Ph.D. Thesis, University of Munich and Università degli Studi dell' Insubria (2004), available at http://edoc.ub.uni-muenchen.de/archive/00001687/

[10] see e.g. D. K. Wójcik and J. R. Dorfman, Phys. Rev. Lett. 90, 230602 (2003); T. A. Brun, H. A. Carteret, and A. Ambainis, ibid. 91, 130602 (2003).

[11] G. Duffy et al., Phys. Rev. E, 70, 056206 (2004); L. Deng et al., Phys. Rev. Lett., 83(26), 5407 (1999) .

[12] A.L. Lichtenberg and M.A. Lieberman, Regular and Chaotic Dynamics, Springer, Berlin, (1992)

[13] M. K. Oberthaler et al., Phys. Rev. Lett. 83, 4447 (1999); S. Fishman, I. Guarneri, and L. Rebuzzini, ibid. 89, 084101 (2002).

[14] R. Graham, M. Schlautmann, and P. Zoller, Phys. Rev. A 45, R19 (1992).

[15] C. Monroe et al., Phys. Rev. Lett. 65, 1571 (1990)

[16] M. Sadgrove et al., Phys. Rev E 71, 027201 (2005). 